carbapenem resistance (i.e. case 2). The occurrence of these resistance mechanisms in immunocompromised cancer patients who have extensive healthcare and antibiotic exposure as well as hospital admission episodes increases the risk of infection spread in healthcare settings as well as in community (case 1). The second case shows that this resistance phenomenon can be found even in children. These cases are, to the best of our knowledge, the first reports of such infections from eastern India. Because of the proximity of this region to highly populous areas and to international trade, tourism, and travel, the likelihood of international dissemination may be high. Surveillance of $\mathrm{mcr}$-mediated colistin resistance may reveal more cases and facilitate better infection control.

Supplementary material. To view supplementary material for this article, please visit https://doi.org/10.1017/ice.2019.363

Acknowledgments. We thank the Indian Council of Medical Research for supporting the study.

Financial support. This study was supported by the Indian Council of Medical Research.

Conflicts of interest. All authors report no conflicts of interest relevant to this article.

\section{References}

1. Liu YY, Wang Y, Walsh TR, et al. Emergence of plasmid-mediated colistin resistance mechanism $m c r-1$ in animals and human beings in China: a microbiological and molecular biological study. Lancet Infect Dis 2016;16:161-168.

2. Aghapour Z, Gholizadeh P, Ganbarov K, et al. Molecular mechanisms related to colistin resistance in Enterobacteriaceae. Infect Drug Resist 2019;12:965-975.

3. Singh S, Pathak A, Kumar A, et al. Emergence of chromosome-borne colistin resistance gene $m c r-1$ in clinical isolates of Klebsiella pneumoniae from India. Antimicrob Agents Chemother 2018;62:pii:e01885-17.

4. Pragasam AK, Shankar C, Veeraraghavan B, et al. Molecular mechanisms of colistin resistance in Klebsiella pneumoniae-causing bacteremia from India—a first report. Front Microbiol 2017;7:2135-2143.

5. Poirel L, Jayol A, Nordmann P. Polymyxins: antibacterial activity, susceptibility testing, and resistance mechanisms encoded by plasmids or chromosomes. Clin Microbiol Rev 2017;30:557-596.

6. Du H, Chen L, Tang YW, Kreiswirth BN. Emergence of the mcr-1 colistin resistance gene in carbapenem-resistant Enterobacteriaceae. Lancet Infect Dis 2016;16:287-288.

7. Xavier BB, Lammens C, Ruhal R et al. Identification of a novel plasmidmediated colistin-resistance gene, mcr-2, in Escherichia coli, Belgium, June 2016. Euro Surveill 2016;21:30280.

8. Yin W, Li H, Shen Y, et al. Novel plasmid-mediated colistin resistance gene mcr-3 in Escherichia coli. MBio 2017;8:pii:e00543-17.

9. Carattoli A, Villa L, Feudi C, et al. Novel plasmid-mediated colistin resistance $m c r-4$ gene in Salmonella and Escherichia coli, Italy 2013, Spain and Belgium, 2015 to 2016. Euro Surveill 2017;22. pii: 30589.

10. Borowiak M, Fischer J, Hammerl JA, Hendriksen RS, Szabo I, Malorny B. Identification of a novel transposon-associated phosphoethanolamine transferase gene, $m c r-5$, conferring colistin resistance in d-tartrate fermenting Salmonella enterica subsp. enterica serovar Paratyphi B. J Antimicrob Chemother 2017;72:3317-3324.

\title{
Zagros Mountains: A region in Iran with extremely high incidence of Brucellosis
}

\author{
Milad Abdi $\mathrm{PhD}^{1,2}$, Rasoul Mirzaei $\mathrm{PhD}^{3}$, Vahid Lohrasbi $\mathrm{PhD}^{2}$ and Khosrow Zamani $\mathrm{PhD}^{2}$ \\ ${ }^{1}$ Student Research Committee, Faculty of Medicine, Iran University of Medical Sciences, Tehran, Iran, ${ }^{2}$ Department of Microbiology, Faculty of Medicine, \\ Iran University of Medical Sciences, Tehran, Iran and ${ }^{3}$ Department of Medical Microbiology, School of Medicine, Hamadan University of Medical Sciences, \\ Hamadan, Iran
}

To the Editor-Brucellosis, or Mediterranean fever, is an important zoonotic disease caused by various species of the gramnegative bacteria Brucella. The well-known species of Brucella are B. abortus, B. melitensis, B. ovis, B. canis, and B. suis, which infect cows, goats, sheep, dogs, and pigs, respectively. ${ }^{1}$ This infectious disease can be transmitted from infected animals to humans through direct contact, tissues (placenta or aborted tissues), or animal products (dairy products). Human to human transmission is rare. ${ }^{2}$ Brucellosis imposes considerable economic losses in many countries each year via damage to livestock through reduced fertility, abortion, and decline in milk production and via humans through significant loss of work days of infected persons and their families and long-term treatment costs. ${ }^{3}$ According to the World Health Organization, $>500,000$ new cases of brucellosis

Author for correspondence: Milad Abdi, Department of Microbiology, Faculty of Medicine, Iran University of Medical Sciences, Tehran, Iran. E-mail: miladabdi1369@ gmail.com

Cite this article: Abdi M, et al. (2020). Zagros Mountains: A region in Iran with extremely high incidence of Brucellosis. Infection Control \& Hospital Epidemiology, 41: 380-382, https://doi.org/10.1017/ice.2019.378 are reported annually worldwide; however, undetected cases are estimated to be $4 \times$ this number. ${ }^{4,5}$ The incidence rate of this infection varies from $<0.01$ to $>200$ cases per 100,000 population in different parts of the world, depending on geographical area, hygiene level, eating habits, occupation, and other factors. ${ }^{6}$ Notably, the incidence rate of this disease (per 100,000 population) is very low in many countries: the United States (0.04), Italy (0.9), Spain (1.3), Russia (0.4), Chile (0.006) France (0.05), South Korea $(0.1)$, and China (0.4). It has even been eradicated in some countries: Sweden, Denmark, Finland, Germany, the United Kingdom (excluding Northern Ireland), Austria, Netherlands, Belgium, Luxembourg, Norway, and Switzerland. Unfortunately, the infection is still endemic in Middle East countries, where high incidence rates (per 100,000 population) prevail: Iran (23.8), Turkey (26.2), Syria (160.3), Saudi Arabia (21.4), and Iraq (27.8). ${ }^{7}$ In Iran, $\sim 16,000$ cases of brucellosis are reported annually. ${ }^{8,9}$ According to data from the National Commission on Communicable Diseases Control, in 1989, the annual incidence exceeded 100 cases per 100,000 population, but in 2013 , the annual incidence had fallen to 23.8 cases per 100,000 population. Despite this decline, 
human brucellosis remains an important health issue in Iran. ${ }^{7}$ Distribution of the disease is uneven across the country, and although cases of the disease have been reported in all provinces of the country, the incidence is higher in the western and northwestern provinces, in the Zagros Mountain region. ${ }^{10}$ In an analytical study in 2011, Pakzad et $\mathrm{al}^{10}$ reported the highest incidence rate of brucellosis in Coohrang County of ChaharmahalBakhtiari province, with 317 cases per 100,000 population. In subsequent years, Charuymaq County of East Azerbaijan province had rates of 384 cases per 100,000 population in 2012, 534 in 2013, and 583 in 2014, which was at most 14575 and 25 times higher than the average incidence rate of brucellosis in the USA and Iran, respectively. ${ }^{10}$ In 2012, Almasi et $\mathrm{al}^{11}$ also reported that the incidence rate of brucellosis in Kermanshah is $\sim 275$ per 100,000 cases, and in 2009 , Bokaie et $\mathrm{al}^{12}$ reported that the incidence rate in Western Azerbaijan was $\sim 175$ per 100,000. In Hamadan, the incidence rate has been reported to be 130 per 100,000 cases. In provinces outside the Zagros Mountains, such as Qom and Tehran, the incidences rate were 7 and 10 per 100,000 cases, respectively. ${ }^{9}$ Overall, the incidence rate of brucellosis in the Zagros Mountains is significantly higher than other provinces. This region may be also related to high incidence rates of brucellosis in Iran, Iraq, and Turkey. Thus, we investigated the factors that affect the rate of brucellosis in the Zagros Mountains to recommend ways to reduce the prevalence of this disease.

The Zagros Mountains, with a total length of 1,600 km, spread across Iran, Iraq, and Turkey. They begin in northwestern of Iran, continue along i western borders, and end at the strait of Hormuz. They encompass the provinces of West Azerbaijan, Lorestan, Hamadan, Markazi, Isfahan, Fars, Kurdistan, Kermanshah, Ilam, Kohgiluyeh and Boyerahmad, Chaharmahal and Bakhtiari, Khuzestan and Kerman, Bushehr and Hormozgan. ${ }^{13}$ The earliest agriculture and animal husbandry began here thousands of years ago in Susa and Anshan, the ancient cities located in these mountains. ${ }^{14}$ The people of the Zagros Mountains have been sheep and goat (boazer goat) herders since $\sim 10,000$ B.C.E. The Zagros people have always been in contact with animals and livestock through hunting and migratory or rural life; therefore, they have continually been at risk for zoonotic diseases. ${ }^{15}$

The high incidence of brucellosis in this area has many causes. ${ }^{5,16-19}$ Several studies have reported a higher density of livestock in these areas. The Zagros region has $\sim 43 \%$ of the total population ( $>34$ million people) of Iran, and it is home to $52 \%$ of all livestock in the country, including cattle, sheep, and goats. Most of the country's nomadic and rural population resides in the Zagros Mountains, with close and continual contact with animals. These residents often assist in animal deliveries and come into contact with placenta and related discharges, which are the most potential agents for transmission of Brucella bacteria. These nomadic areas have a low level of hygiene and lack of access to antibacterial detergents and disinfectants. The population in these rural areas, especially nomads, lack sufficient education regarding transmission, manifestation, and complications of brucellosis. They have high and continuous consumption of nonpasteurized dairy products, and they consume raw and semi-raw dairy products (eg, milk and cream). Due to continued movement and living in areas with difficult accessibility, monitoring and vaccination of nomad herds is very difficult and not very effective. Often, several nomads come together in one place, causing cross transmission of Brucella from one herd to another. Transmission also occurs from livestock to wild animals living in the Zagros Mountains, which makes them reservoirs of infection. Other reasons for the high brucellosis rate include unauthorized animal trafficking from neighboring countries and lack of a quarantine system at the borders; lack of appropriate identification system for infectious disease in livestock; traditional, unauthorized, and unsanitary slaughter of animals; and lack of funds.

Despite all these obstacles, we believe that reducing or even eradicating this infection in the Zagros region and more generally in Iran in the long term is possible. We need more effective plans, efforts, cooperation, and especially aid from international organizations such as WHO. Education is a low-cost but very effective measure that increases awareness of and information about brucellosis, its transmission, complications, manifestations, and risk factors. Personal hygiene when assisting animal delivery, boiling milk, and disuse of raw dairy products are other areas of education that can be relatively easily accomplished. Allocating free or cheap drug and health supplies to aid with hygiene can help reduce this infection in nomadic and rural areas. Monitoring and conducting regular vaccination of livestock, preventing animal trafficking, and creating a quarantine system at the borders to identify sick animals are other examples of appropriate measures that will contribute to achieving this valuable goal. ${ }^{4}$

\section{Acknowledgments. None.}

Financial support. No financial support was provided relevant to this article.

Conflicts of interest. All authors report no conflicts of interest relevant to this article.

\section{References}

1. Olsen S, Palmer M. Advancement of knowledge of Brucella over the past 50 years. Vet Path 2014;51:1076-1089.

2. Köse Ş, Senger SS, Akkoçlu G, et al. Clinical manifestations, complications, and treatment of brucellosis: evaluation of 72 cases. Turk J Med Sci 2014;44:220-223.

3. McDermott J, Grace D, Zinsstag J. Economics of brucellosis impact and control in low-income countries. Rev Sci Tech 2013;32:249-261.

4. Asiimwe BB, Kansiime C, Rwego IB. Risk factors for human brucellosis in agro-pastoralist communities of south western Uganda: a case-control study. BMC Res Notes 2015;8:405.

5. Corbel MJ. Brucellosis in Humans and Animals. Geneva: World Health Organization; 2006.

6. Al-Anazi K, Al-Jasser A. Brucellosis: a global re-emerging zoonosis history, epidemiology, microbiology, immunology and genetics. Saudi Arabia: OMICS Group International; 2013.

7. Pappas G, Papadimitriou P, Akritidis N, Christou L, Tsianos EV. The new global map of human brucellosis. Lancet Infect Dis 2006;6:91-99.

8. Pakzad I, Hosseinzadehgan $\mathrm{H}$, Abtahi $\mathrm{H}$, et al. Human brucellosis in Iran: incidence, complication, diagnosis, treatment and prevention. J Pure Appl Microbiol 2013;7:1177-1188.

9. Mirnejad R, Jazi FM, Mostafaei S, Sedighi M. Epidemiology of brucellosis in Iran: a comprehensive systematic review and meta-analysis study. Microb Pathogen 2017;109:239-247.

10. Pakzad R, Pakzad I, Safiri S, et al. Spatiotemporal analysis of brucellosis incidence in Iran from 2011 to 2014 using GIS. Int J Infect Dis 2018;67:129-136.

11. Almasi A, Hashemian A, Azizi A, et al. Epidemiology of brucellosis in population under coverage of Mahidasht health and Curative center through 2004-2010 based on Geographical Information System (GIS). Archives Des Sciences 2010;65:406-412.

12. Bokaei S, Heydari LS, Abbaszadeh S, et al. Ecological study of brucellosis in humans and animals in Khoy, a mountainous District of the IR. of Iran. Iran J Microbiol 2009;1:14-17.

13. State divisions in Iran. Statistical Center of Iran website. https://www.amar. org.ir/english/Iran-at-a-glance/Iran. Published 2019. Accessed December 30, 2019. 
14. Bahmanimehr A, Nikmanesh F, Yepiskoposyan L. Paternal genetic landscape of Zagros region of Iran and its role in the gene flow in the populations of the region. Iran J Epidemiol 2014;10(3):43-53.

15. Vigne J-D. The origins of animal domestication and husbandry: a major change in the history of humanity and the biosphere. Comptes rendus biologies 2011;334:171-181.

16. Esmaeili H. Brucellosis in the Islamic republic of Iran. J Med Bacteriol 2014;3(3-4):47-57.
17. Honarvar B, Moghadami M, Lankarani K, et al. Brucellosis as a neglected disease in a neglected population: a seroepidemiological study of migratory nomads in the Fars province of Iran. Epidemiol Infect 2017;145:491-497.

18. Franc K, Krecek R, Häsler B, Arenas-Gamboa A. Brucellosis remains a neglected disease in the developing world: a call for interdisciplinary action. BMC Pub Health 2018;18:125.

19. Agriculture. Statistical Center of Iran website. https://www.amar.org.ir/ english/Statistics-by-Topic/Agriculture\#113281-releases. Published 2019. Accessed December 30, 2019.

\title{
Sustaining outpatient antimicrobial stewardship: Do we need to think further outside the box?
}

\author{
Holly M. Frost MD ${ }^{1,2}$ (D), Leisha M. Andersen MD, MPH ${ }^{3}$, Katherine E. Fleming-Dutra MD², Chuck Norlin $\mathrm{MD}^{5}$ and \\ Christopher A. Czaja MD, MPH ${ }^{6}$ \\ ${ }^{1}$ Department of Pediatrics, Denver Health Medical Center, Denver, Colorado, ${ }^{2}$ Department of Pediatrics, University of Colorado School of Medicine, Aurora, \\ Colorado, ${ }^{3}$ American Academy of Pediatrics- Colorado Chapter, Denver, Colorado, ${ }^{4}$ Division of Healthcare Quality Promotion, Centers for Disease Control and \\ Prevention, Atlanta, Georgia, ${ }^{5}$ Department of Pediatrics, University of Utah, Salt Lake City, Utah and ${ }^{6}$ Colorado Department of Public Health and Environment, \\ Denver, Colorado
}

To the Editor-Antibiotic overuse is a persistent public health problem that has contributed to an alarming increase in the prevalence of antibiotic resistance. ${ }^{1}$ In the United States, $>70 \%$ of antibiotic courses are prescribed in ambulatory settings. ${ }^{2}$ To encourage the appropriate use of antibiotics in outpatient practices, the Centers for Disease Control and Prevention (CDC) released the Core Elements of Outpatient Antibiotic Stewardship (hereafter Core Elements). ${ }^{3}$ However, detailed evaluations of how practices implement and sustain the Core Elements are lacking.

From January through June 2019, an antimicrobial stewardship quality improvement initiative was conducted by the American Academy of Pediatrics Chapter Quality Network (AAP-CQN), Colorado Department of Public Health and Environment, and the CDC. In this project, 8 outpatient pediatric practices in Colorado were enrolled: 3 practices that were part of larger systems and 5 independent practices. All practices but 1 were urban or suburban. The initiative utilized the Core Elements and Model for Improvement frameworks. ${ }^{3}$ Practices implemented interventions to improve prescribing for pharyngitis and acute otitis media. The AAP-CQN provided webinars, learning sessions, monthly tracking and reporting, and Maintenance of Certification (MOC) credit. The ability of the practices to continue antibiotic stewardship following the conclusion of the initiative was assessed.

Using surveys and telephone-based semistructured interviews with physician champions, we assessed the ability of practices to sustain the Core Elements of commitment, tracking and reporting, and education and expertise. Because the initial intervention focused on evaluation of the Core Element "action for policy and practice," our interviews targeted the other Core Elements. The project was determined exempt by the AAP Institutional Review Board.

Author for correspondence: Holly M. Frost, E-mail: Holly.frost@dhha.org

Cite this article: Frost HM, et al. (2020). Sustaining outpatient antimicrobial stewardship: Do we need to think further outside the box?. Infection Control \& Hospital Epidemiology, 41: 382-384, https://doi.org/10.1017/ice.2019.366
Most practices identified a leader for antibiotic stewardship (7 of 8) and intended to display commitment posters (5 of 7). ${ }^{4}$ Most had access to antibiotic expertise (6 of 8) and planned to provide ongoing education regarding antibiotic use (6 of 8). Few practices had established systems for ongoing antibiotic stewardship-related duties (4 of 8), tracking (5 of 8), or reporting (3 of 7).

Interviews identified 6 barriers to sustaining the 3 Core Elements (Table 1). Lack of control and competing priorities complicated practices' capacity to display commitment. Of the 5 practices reporting implementation of a commitment poster on the survey, only 2 affirmed the finding during interviews. Barriers to displaying posters included modifying the CDC template, inserting provider photos, and printing logistics. For practices that were part of a larger healthcare system, the need to obtain administrative approval (for information technology (IT) support or to hang commitment posters) was a key limitation.

Barriers to tracking and reporting included time constraints and lack of information technology (IT) support. Only 2 practices had IT support. Practices reported that manual or electronic abstraction took substantial time (range, $4-20$ hours by a physician or clinic manager). Consequently, 5 practices did not plan to continue tracking and reporting, and 3 practices planned abbreviated and less frequent (every 3-6 months) tracking and reporting. Practices were most likely to sustain tracking and reporting if a nonphysician was responsible for data.

Barriers to sustaining education/expertise included scheduling and lack of resources (eg, regional experts). In the absence of MOC and outside support, the structures of most practices did not support scheduled education on antibiotic stewardship.

To sustain antibiotic stewardship, practices would benefit from increased availability of practical strategies and tools. Strategies to support the commitment to the Core Elements might include a centralized online system for practices to design and order custom commitment posters. For our initiative, AAP-Colorado created and supplied posters, which were well received. Because tracking and 